\title{
Time propagation of the coupled Maxwell and Kohn-Sham equations using the Riemann-Silberstein formalism
}

\author{
Cody Covington* \\ Department of Physics and Astronomy, Vanderbilt University, Nashville, Tennessee 37235, USA \\ and Department of Chemistry, Austin Peay State University, Clarksville, Tennessee 37044, USA \\ Daniel Kidd ${ }^{\dagger}$ and Haley Buckner ${ }^{0}$ \\ Department of Physics and Astronomy, Vanderbilt University, Nashville, Tennessee 37235, USA
}

Heiko Appel

Max Planck Institute for the Structure and Dynamics of Matter, Center for Free Electron Laser Science, 22761 Hamburg, Germany

Kálmán Varga $\oplus^{*}$

Department of Physics and Astronomy, Vanderbilt University, Nashville, Tennessee 37235, USA

(Received 10 July 2019; published 4 November 2019)

\begin{abstract}
The coupled Maxwell and time-dependent Kohn-Sham equations are solved using the Riemann-Silberstein vectors to represent the electromagnetic fields. Momentum-space time propagation of the Riemann-Silberstein vectors are proposed and test calculations are presented to show the accuracy of the approach.
\end{abstract}

DOI: 10.1103/PhysRevE.100.053301

\section{INTRODUCTION}

The conversion of electromagnetic (EM) waves into collective excitations of electrons [1,2], a cross fertilization between optics, material science, and electronics, is an overarching theme among a wide range of important applications in nanophotonics [3,4], biology [5], sensing [6], singlemolecule detection [7], nanophotonic lasers [8], photovoltaic devices $[9,10]$, spectroscopy $[11,12]$, and solar energy harvesting $[13,14]$. Computational electrodynamics modeling, that is the simulated propagation of EM waves in matter using the Maxwell equations, has been an invaluable tool in the study and design of new materials.

The most popular approach for simulating the electromagnetic response of materials to the propagation of Maxwell's equations in space and time is the finite-difference timedomain (FDTD) method $[15,16]$. The FDTD method is considered to be stable and reliable for most materials where the optical properties are known. The FDTD method, however, relies on macroscopic material properties, namely the permeability, permittivity, and conductivity of each point in space, thus limiting the applicability of the method. Furthermore, it is not easily applied to the modeling of surfaces, interfaces, and nonlinear materials.

By the constant miniaturization of electronic and optical devices towards the nanoscale, electromagnetic simulations inevitably confront the quantum regime [17-22] where classical approaches based on macroscopic material properties fail. The importance of quantum effects in strongly coupled

\footnotetext{
*cody.1.covington@ vanderbilt.edu

†daniel.w.kidd@vanderbilt.edu

*kalman.varga@vanderbilt.edu
}

light matter systems, e.g., nanoplasmonics and nano-optics, have attracted intense experimental interest in the last few years $[19,23,24]$. These experiments are able to probe the local electronic states [23], the Raman scattering images of molecular structures [25], and the plasmon couplings [24] at the nanometer length-scale.

The importance of quantum effects has been realized by the computational electromagnetics community, and conventional finite-difference time-domain electromagnetic simulations have been complemented with a coupling to the Schrödinger equation [26-29].

However, time-dependent quantum mechanical simulations have also started to incorporate electromagnetic waves to describe the light matter interaction [30-37]. Most of these calculations use the EM waves as external driving fields, but induced fields [31,38] and propagation of EM waves in a multiscale manner [39] have also been investigated. Recently, extensions of time-dependent density functional theory have also been proposed to treat light and matter simultaneously on a quantized level [40,41]. The different levels of computational approaches have been used to describe laser irradiation [42], dielectric response [43,44] high harmonic generation in solids [45,46], the dynamical Franz-Keldysh effect [47], attosecond band gap dynamics [48], local currents [49,50], Rabi splittings of infrared spectra for molecules in optical cavities [51], modification of excitation and charge transfer in optical cavities [52], photoemission [53], and Coulomb explosion as well as ionization [54,55].

The integration of a quantum description of electrons with the propagation of EM waves for a description of the interaction of light and matter has been attempted at various levels of approximation. Computational approaches have to address many challenges arising from the different time and length scales used within the propagation of the EM waves and 
electron wave functions. On the theory level, one has to find an acceptable compromise between the fully microscopic quantum description of both electrons and photons [56,57] and the macroscopic and/or classical field treatment of the EM waves. The most rigorous approach, the quantum electrodynamical density functional theory [40,58-61], is a framework which allows to describe quantum particles interacting with a quantized electromagnetic field. While with this approach such a level of description becomes computationally feasible, a full implementation of the method for extended systems is in progress but has not been reached so far. On a different level of approximation, the quantum mechanical system of electrons is coupled to classical EM fields, and the problem of length scales is bridged using multiscale approaches [57,62-64].

Coupled Maxwell-Schrödinger approaches based on the FDTD method have also been developed. These approaches neglect atomistic details and electron correlations, being primarily interested in the propagation of EM waves in the presence of matter within large systems [28,29,65-69]. Various gauges [68,69] and unified frameworks [65] have been proposed and tested to make the calculations efficient. In these approaches the Maxwell equations are solved using the FDTD method on a Yee lattice [70] with appropriate boundary conditions [71-73]. Once the electric and magnetic fields are calculated, the corresponding vector potential is plugged into the Schrödinger equation. The Schrödinger equation is solved in the following step, and the polarization current density is then the subsequent determined from the wave function. This polarization current density is then input into of the Maxwell equations.

For EM waves the FDTD algorithm is rather fast and can be done numerous times in succession without significant computational expense or difficulty. There is, however, an inherent problem with time propagating the EM waves and electrons using the same spatial dimensions. EM waves travel much faster than electron waves and have much longer wavelengths. If the same 3D grid is used to discretize both EM and electron waves, the time propagation of EM fields will require very short time steps. The grid spacing and time steps are constrained by the Courant condition, that is $c \Delta t / \Delta x<1$, connecting the speed of light $\mathrm{c}$, the time step $\Delta t$ and the spatial discretization $\Delta x$. This seriously limits the flexibility when plugging the electromagnetic vector potential into the Schrödinger equation. Furthermore, the small grid spacings and rapidly changing current densities required in quantum mechanical calculations may lead to instabilities in the FDTD method that can cause catastrophic failures.

In the simulations described above, the FDTD method has been used to propagate both the EM waves and the electron wave function-that is, the the Schrödinger equation has been incorporated within the EM waves propagation framework [27-29,62,66]. In this paper we take the opposite approach wherein the Maxwell dynamics will be incorporated into the well developed quantum mechanical wavefunction propagation framework. For the quantum mechanical description of electron dynamics, the time-dependent density functional theory (TDDFT) [74] is the method of choice due to its balance of computational efficiency and accuracy. The challenge is then to couple Maxwell's equations with the timedependent Schrödinger (Kohn-Sham) equation through the current density produced by the electrons and the EM fields. For simultaneous time propagation of the electromagnetic waves and electronic wave functions in a quantum framework we will use in the present work the Maxwell wave-function approach.

The Maxwell wave-function formalism in connection with the time propagation of the coupled Maxwell-TDDFT equations was first proposed in Ref. [61]. This work establishes the theoretical foundations of a density functional approach for coupled photons, electrons, and effective nuclei in nonrelativistic QED.

In the Maxwell wave-function approach, the Maxwell equations are recasted into a time-dependent Schrödinger-like equation using the Riemann-Silberstein (RS) vector formalism [75]. The RS vector describes both electric and magnetic fields as a single complex quantity. With this combination, a single time-evolution operator can be constructed instead of using two coupled differential equations-Maxwell's curl equations. Furthermore, the time-evolution can be done analytically using the momentum representation [76], and conversion between momentum and spatial representations can be done by fast-Fourier transforms (FFTs). The advantage of this approach is that the same time propagation framework that is typically used for matter dynamic $[38,76,77]$ now can be used for both the Maxwell and quantum equations.

In Ref. [61], the RS vector was propagated using finitedifference representation and the boundary conditions have been enforced by the Perfectly Matched Layers approach [16]. In this work we explore an alternative implementation: momentum-space propagation of the RS vector and complex absorbing potentials to avoid reflections from the boundary. As using the RS formalism to construct the Maxwell-TDDFT equations is a new approach, various implementations must be tested to find the most suitable and efficient method.

The paper is organized as follows. In Sec. II we introduce the theoretical method. In Sec. III various simulations are performed using jellium systems. Finally, in Sec. IV the paper is closed with a summary and future outlook.

\section{FORMALISM}

In the following we describe the formalism for both the RS vector approach to the Maxwell equations and TDDFT.

\section{A. The RS vector and Maxwell's Equations}

Maxwell's equations are known to describe electromagnetic radiation and are given as

$$
\begin{gathered}
\nabla \cdot \mathbf{E}=\frac{\rho}{\epsilon_{0}}, \quad \nabla \cdot \mathbf{B}=0 \\
\nabla \times \mathbf{B}=\frac{1}{c^{2}} \frac{\partial \mathbf{E}}{\partial t}+\mu_{0} \mathbf{J}, \quad \nabla \times \mathbf{E}=-\frac{\partial \mathbf{B}}{\partial t},
\end{gathered}
$$

where $\mathbf{E}$ and $\mathbf{B}$ are the electric and magnetic fields, $\rho$ and $\mathbf{J}$ are the charge and current densities, $\epsilon_{0}$ and $\mu_{0}$ are the vacuum permittivity and permeability, and $c=\left(\epsilon_{0} \mu_{0}\right)^{-1 / 2}$ is the speed of light.

The Riemann-Silberstein (RS) vector [75] is constructed as

$$
\mathbf{F}(\mathbf{r}, t)=\sqrt{\frac{\epsilon_{0}}{2}} \mathbf{E}(\mathbf{r}, t) \pm i \sqrt{\frac{1}{2 \mu_{0}}} \mathbf{B}(\mathbf{r}, t) .
$$


The sign of the imaginary part of the Riemann-Silberstein vector corresponds to different helicities. Equation (1) may now be rewritten as

$$
\nabla \cdot \mathbf{F}=\frac{1}{\sqrt{2 \epsilon_{0}}} \rho .
$$

In the same way, Eq. (2) may also be rewritten as

$$
i \hbar \frac{\partial \mathbf{F}}{\partial t}=c\left(\mathbf{S} \cdot \frac{\hbar}{i} \nabla\right) \mathbf{F}-\frac{i \hbar}{\sqrt{2 \epsilon_{0}}} \mathbf{J}
$$

where the elements of $\mathbf{S}=\left(S_{x}, S_{y}, S_{z}\right)$ are the spin 1 counterparts of the Pauli matrices,

$$
\begin{aligned}
S_{x} & =\left(\begin{array}{ccc}
0 & 0 & 0 \\
0 & 0 & -i \\
0 & i & 0
\end{array}\right), \quad S_{y}=\left(\begin{array}{ccc}
0 & 0 & i \\
0 & 0 & 0 \\
-i & 0 & 0
\end{array}\right), \\
S_{z} & =\left(\begin{array}{ccc}
0 & -i & 0 \\
i & 0 & 0 \\
0 & 0 & 0
\end{array}\right) .
\end{aligned}
$$

Equation (5) may be viewed as being analogous to the time-dependent Schrödinger equation, where one has an effective Hamiltonian,

$$
H_{\mathrm{EM}}=c\left(\mathbf{S} \cdot \frac{\hbar}{i} \nabla\right),
$$

acting on three "orbitals"- those being the three dimensional components of the RS vector. There is also a source term $\mathbf{S}(\mathbf{r}, t)=-\frac{i \hbar}{\sqrt{2 \epsilon_{0}}} \mathbf{J}(\mathbf{r}, t)$ which is represented for each "orbital" as well. We therefore may write

$$
i \hbar \frac{\partial}{\partial t} \mathbf{F}(\mathbf{r}, t)=H_{\mathrm{EM}} \mathbf{F}(\mathbf{r}, t)+\mathbf{S}(\mathbf{r}, t)
$$

The solution for this equation using a discretized small-timestep approach is [77]

$$
\begin{aligned}
\mathbf{F}\left(\mathbf{r}, t+\Delta t_{\mathrm{EM}}\right)= & e^{-i H_{\mathrm{EM}} \Delta t_{\mathrm{EM}} / \hbar} \mathbf{F}(\mathbf{r}, t)-\frac{i}{\hbar} e^{-i H_{\mathrm{EM}} \Delta t_{\mathrm{EM}} / \hbar} \\
& \times \int_{0}^{\Delta t_{\mathrm{EM}}} e^{i H_{\mathrm{EM}} \tau / \hbar} \mathbf{S}(\mathbf{r}, t+\tau) d \tau .
\end{aligned}
$$

Here the $\Delta t_{\mathrm{EM}}$ denotes the time step used to advance the RS vector. If the time step is small enough, the the integrand may be assumed to be constant. Then the integral and preceding operator combine resulting in

$$
\mathbf{F}\left(\mathbf{r}, t+\Delta t_{\mathrm{EM}}\right)=e^{-i H_{\mathrm{EM}} \Delta t_{\mathrm{EM}} / \hbar} \mathbf{F}(\mathbf{r}, t)-\frac{i}{\hbar} \mathbf{S}(\mathbf{r}, t) \Delta t_{\mathrm{EM}} .
$$

After plugging back in the definition for $S$, this yields

$$
\mathbf{F}\left(\mathbf{r}, t+\Delta t_{\mathrm{EM}}\right)=e^{-i H_{\mathrm{EM}} \Delta t_{\mathrm{EM}} / \hbar} \mathbf{F}(\mathbf{r}, t)-\frac{1}{\sqrt{2 \epsilon_{0}}} \mathbf{J}(\mathbf{r}, t) \Delta t_{\mathrm{EM}} .
$$

As $H_{\mathrm{EM}}$ is time independent, the exponential propagator for $\mathbf{F}(\mathbf{r}, t)$ is exact for any time step [77]. The time-step error is caused by the approximation of the integral in Eq. (10), which in our case is second order in $\Delta t_{\mathrm{EM}}$. The time propagation of a Schrödinger-like equation with a source term has been investigated in Ref. [78] where higher-order approximations with smaller time-step errors have been developed. A more detailed discussion of the time propagation of the $\mathrm{RS}$ vector can be found in Ref. [61].

By casting the differential equation as a time-evolution operator, one can use the same time propagation scheme for the EM waves and the electron wave functions providing a unified framework. In particular, FFT can be used to transform $\mathbf{F}(\mathbf{r}, t)$ into momentum-space,

$$
\tilde{\mathbf{F}}(\mathbf{k}, t)=\mathcal{F}[\mathbf{F}(\mathbf{r}, t)],
$$

where the $e^{-i H_{\mathrm{EM}} \Delta t / \hbar}$ operator is diagonal; its action can be easily calculated using element-wise multiplication, and then an inverse FFT may be used to return the result to a real-space representation:

$$
\mathbf{F}(\mathbf{r}, t)=\mathcal{F}^{-1}[\tilde{\mathbf{F}}(\mathbf{k}, t)] .
$$

The time evolution of the RS vector is easily performed in Fourier space using the Fourier representation of the RS vector and the Fourier representation of the current, given as

$$
\tilde{\mathbf{F}}\left(\mathbf{k}, t+\Delta t_{\mathrm{EM}}\right)=e^{-i H_{\mathrm{EM}} \Delta t_{\mathrm{EM}} / \hbar} \tilde{\mathbf{F}}(\mathbf{k}, t)-\frac{1}{\sqrt{2 \epsilon_{0}}} \tilde{\mathbf{J}}(\mathbf{k}, t) \Delta t_{\mathrm{EM}} .
$$

The advancement in time of the RS vector defines the time evolution operator, written as

$$
\tilde{\mathbf{F}}(\mathbf{k}, t+\delta t)=\mathcal{R}(t, t+\delta t) \tilde{\mathbf{F}}(\mathbf{k}, t) .
$$

where time $t+\delta t$ is reached by successive applications of Eq. (14). In this way the time evolution of the RS vector proceeds analytically on the Fourier components, until its real space representation is needed, at which time an inverse FFT is used.

It should also be noted that in the equations given above, the same time index $t$ is given for $\mathbf{F}$ and $\mathbf{J}$; however, some advanced time-integration schemes may occur where the explicit time may differ between the two.

To couple the Maxwell equations to the Schrödinger equation, we need the vector potential $\mathbf{A}(\mathbf{r}, t)$. To calculate the vector potential from the electric field we split the electric field as

$$
\mathbf{E}(\mathbf{r}, t)=\mathbf{E}_{\mathrm{rot}}(\mathbf{r}, t)+\mathbf{E}_{\mathrm{irr}}(\mathbf{r}, t)
$$

so that

$$
\nabla \cdot \mathbf{E}_{\mathrm{rot}}(\mathbf{r}, t)=0, \quad \nabla \times \mathbf{E}_{\mathrm{irr}}(\mathbf{r}, t)=0 .
$$

Vector fields meeting these criteria can be obtained through a Helmholtz decomposition (HD), which can be performed trivially on the Fourier components of $\mathbf{F}(\mathbf{r}, t)$.

Using the Coulomb gauge, which requires

$$
\nabla \cdot \mathbf{A}(\mathbf{r}, t)=0
$$

the vector potential can be calculated as

$$
\mathbf{A}(\mathbf{r}, t)=-\int_{\tau=0}^{t} \mathbf{E}_{\mathrm{rot}}(\mathbf{r}, \tau) d \tau .
$$

An external vector potential can be added to the solenoidal part of the electric field (calculated from the RS vector),

$$
\mathbf{A}(\mathbf{r}, t)=-\int_{\tau=0}^{t} \mathbf{E}_{\mathrm{rot}}(\mathbf{r}, \tau)+\mathbf{E}_{\mathrm{Ext}}(\mathbf{r}, \tau) d \tau .
$$


This is only possible if the entire simulation cell has the same relative permittivity and permeability. Otherwise, a source must be introduced that generates an incident RS vector corresponding to the desired external field. Alternatively, for short enough pulses that fit entirely within the bounding box, the RS vector may be initialized using the external fields.

We note that the use of a HD allows for some options for the calculation of the scalar potential. The scalar potential calculated from $\mathbf{E}_{\mathrm{irr}}(\mathbf{r}, t)$ can be added to the potential at the beginning of the simulation, and in this way, the Hartree potential can be obtained. However, the potential is obtained from electron currents introduced to the Maxwell equations and not from the electron density directly. We therefore calculate the Hartree potential by solving the Poisson equation using the electron density.

\section{B. Absorbing Boundary Conditions for the RS Method}

Since the RS method utilizes the Fourier representation, it is periodic by definition. This is somewhat of a disadvantage when compared to FD methods, where periodicity is optional. To make simulations using the RS method finite, absorbing boundary conditions are required. Typical absorbing boundary conditions are not simply applied to the RS vector propagated in this way, because the derivatives of $\mathbf{F}$ are not easily obtained at the boundaries while the time evolution is being applied, since the exponential is applied to the Fourier components.

A simpler alternative is to use an absorbing function in real-space, akin to the way the electronic wave function is prevented from reflecting from the boundaries using complex absorbing potentials (CAP) [79,80]. In the CAP approach, an imaginary potential $i W_{\mathrm{EM}}(\mathbf{r})$, usually a polynomial function, is added to the Hamiltonian,

$$
H_{\mathrm{EM}}(\mathbf{r}, t)+i W_{\mathrm{EM}}(\mathbf{r}),
$$

and the new Hamiltonian is time propagated. The potential is only nonzero outside of the physical region where it absorbs outgoing waves. The following functional form was chosen for the RS CAP:

$$
W_{\mathrm{EM}}(\mathbf{r})=-2 \pi^{2}\left(c_{1} h+c_{2} h^{2}+c_{3} h^{3}+c_{4} h^{4}\right) .
$$

Here, $h=10\left(x-x_{0}\right) / l, x_{0}$ is the end of the physical region, $x_{0} \leqslant x \leqslant x_{0}+l$, and $l$ is the width of the absorbing boundary. We note that $l$ should encompass at least 15 grid points. While this defines CAPs in the $x$ direction, CAPs may be added in other directions as needed. Coefficients for the polynomial were obtained by a mixed genetic-gradient optimization algorithm resulting in values of $c_{1}=1.27967 \times 10^{-3}, c_{2}=$ $4.86973 \times 10^{-4}, c_{3}=9.78732 \times 10^{-3}$, and $c_{4}=2.77563 \times$ $10^{-4}$, each given here in atomic units. CAPs employing these coefficients exhibited a typical reduction by 4-7 orders of magnitude in the reflected electric field.

Incorporating the CAPs modifies the time propagation formalism of the RS vector. The CAP can be efficiently added using the split operator approach, where the exponential is split, separating the potential from the derivative operators.
Using the split operator approach one obtains

$$
\begin{aligned}
\mathbf{F}(\mathbf{r}, t+\Delta t)= & e^{W_{\mathrm{EM}}(\mathbf{r}) \Delta t / 2} \mathcal{F}^{-1}[\mathcal{R}(t, t+\Delta t) \mathcal{F} \\
& \left.\times\left[e^{W_{\mathrm{EM}}(\mathbf{r}) \Delta t / 2} \mathbf{F}(\mathbf{r}, t)\right]\right] .
\end{aligned}
$$

Note that the CAP is diagonal in real-space so the exponentiation does not add to the computational cost.

\section{Kohn-Sham Hamiltonian}

We will use density functional theory (DFT) [81] to initialize the quantum mechanical system and TDDFT [74] to simulate the dynamics of the electrons. The ground-state Kohn-Sham (KS) Hamiltonian, $H_{\mathrm{KS}}$, is given by

$$
H_{\mathrm{KS}}=-\frac{\hbar^{2}}{2 m} \nabla_{\mathbf{r}}^{2}+V_{\mathrm{BG}}(\mathbf{r})+V_{\mathrm{H}}[\rho](\mathbf{r})+V_{\mathrm{XC}}[\rho](\mathbf{r}),
$$

where $\rho$ is the electron density obtained by a sum over all occupied orbitals, $V_{\mathrm{BG}}$ is the background potential, $V_{\mathrm{H}}$ is the Hartree potential, and $V_{\mathrm{XC}}$ is the exchange-correlation potential. $V_{\mathrm{BG}}$ is determined by solving the Poisson equation for a uniform background charge with a prescribed density, corresponding to a jellium model of electrons. In calculations with atomistic details this term would be replaced by an electron-nuclei Coulomb potential using an all-electron or pseudopotential approach. Such approaches are routinely used in DFT and TDDFT calculations and using such here would not interfere with the current formalism. The Hartree potential, $V_{\mathrm{H}}$, is given by

$$
V_{\mathrm{H}}(\mathbf{r})=\int d \mathbf{r}^{\prime} \frac{\rho\left(\mathbf{r}^{\prime}\right)}{\left|\mathbf{r}-\mathbf{r}^{\prime}\right|}
$$

and accounts for the electrostatic Coulomb interaction between electrons. Equation (25) is computed by numerically solving the Poisson equation. To represent the exchangecorrelation potential, $V_{\mathrm{XC}}$, we employ the adiabatic localdensity approximation (ALDA) with the parameterization of Perdew and Zunger [82].

\section{Time propagation of electrons}

To couple the EM fields to the electron dynamics, the kinetic energy operator is replaced-using velocity gauge-in the KS Hamiltonian

$$
\begin{aligned}
H_{\mathrm{KS}}(\mathbf{r}, t)= & -\frac{1}{2 m}\left[-i \hbar \nabla_{\mathbf{r}}+\mathbf{A}(\mathbf{r}, t)\right]^{2} \\
& +V_{\mathrm{BG}}(\mathbf{r})+V_{\mathrm{H}}[\rho](\mathbf{r})+V_{\mathrm{XC}}[\rho](\mathbf{r}) \\
= & -\frac{1}{2 m}\left[-i \hbar \nabla_{\mathbf{r}}+\mathbf{A}(\mathbf{r}, t)\right]^{2}+V_{\mathrm{KS}}(\mathbf{r}, t),
\end{aligned}
$$

and the electron dynamics is governed by the time-dependent $\mathrm{KS}$ equation. The time-dependent KS (TD-KS) equation for the $i$ th electron orbital is

$$
\left(i \hbar \frac{\partial}{\partial t}-H_{\mathrm{KS}}\right) \psi_{i}(\mathbf{r}, t)=0
$$

This equation can be solved by time propagating the wave function from the initial state to some time, $t$, by using the 
time-evolution operator,

$$
\begin{aligned}
& U(0, t)=\mathcal{T} \exp \left[-\frac{i}{\hbar} \int_{0}^{t} H_{\mathrm{KS}}\left(\mathbf{r}, t^{\prime}\right) d t^{\prime}\right], \\
& \psi_{i}(\mathbf{r}, t)=U(0, t) \psi_{i}(\mathbf{r}, 0),
\end{aligned}
$$

where $\mathcal{T}$ denotes time-ordering. In practice, $U(0, t)$ is split into a product of multiple short-time propagators,

$$
U(0, t)=\prod_{q} U\left(t_{q}, t_{q}+\delta t\right), \quad t_{q}=q \delta t,
$$

which evolves the Kohn-Sham orbitals from $t_{q}$ to $t_{q}+\delta t$. The short-time-step propagator is defined by

$$
U\left(t_{q}, t_{q}+\delta t\right)=\exp \left[-\frac{i \delta t}{\hbar} H_{\mathrm{KS}}\left(\mathbf{r}, t_{q}\right)\right] .
$$

The time step, $\delta t$, is chosen to be sufficiently small so that the Hamiltonian can be treated as constant over the course of one step. A fourth-order Taylor expansion is used to approximate Eq. (30),

$$
U\left(t_{q}, t_{q}+\delta t\right) \approx \sum_{n=0}^{4} \frac{1}{n !}\left[-\frac{i \delta t}{\hbar} H_{\mathrm{KS}}\left(\mathbf{r}, t_{q}\right)\right]^{n} .
$$

While the Taylor propagation scheme is conditionally stable, one must choose a suitably small time step to preserve unitarity approximately during the time propagation.

The time dependent electron density and the electron current are defined as

$$
\begin{gathered}
\rho(\mathbf{r}, t)=2 \sum_{i=1}^{N_{e} / 2}\left|\psi_{i}(\mathbf{r}, t)\right|^{2}, \\
\mathbf{J}(\mathbf{r}, t)=2 \operatorname{Re} \sum_{i=1}^{N_{e} / 2}\left[\psi_{i}(\mathbf{r}, t)^{*}\left(-i \hbar \nabla_{\mathbf{r}}+\mathbf{A}(\mathbf{r}, t)\right) \psi_{i}(\mathbf{r}, t)\right],
\end{gathered}
$$

where $N_{e}$ is the number of electrons in the system and each orbital is occupied by two electrons.

In practice, this time step for electrons can be larger than that used during the propagation of the RS vector, $\Delta t_{\mathrm{EM}}$. For the propagation scheme used in this work, where electrons are propagated by Taylor expansion in real-space with finite difference and the RS vector is propagated analytically in momentum-space, $\Delta t_{\mathrm{EM}}$ should be at least 20 times smaller than $\Delta t$.

One additional consideration is that as the electrons are driven to excited states by the vector potential, some electron density will reach the boundary of the simulation cell (see Fig. 1). To avoid the nonphysical wrapping of periodic boundary conditions (PBCs) in the $x$ direction, a CAP of the aforementioned form given by Manolopoulos [80] is added in the region close to the boundary.

\section{E. Combined RS and KS time propagation}

To propagate the RS vectors and KS orbitals one needs to choose a space and time grid. For simplicity and for test purposes, we use the same spatial grid for both propagations. This is obviously not ideal and one can design much more

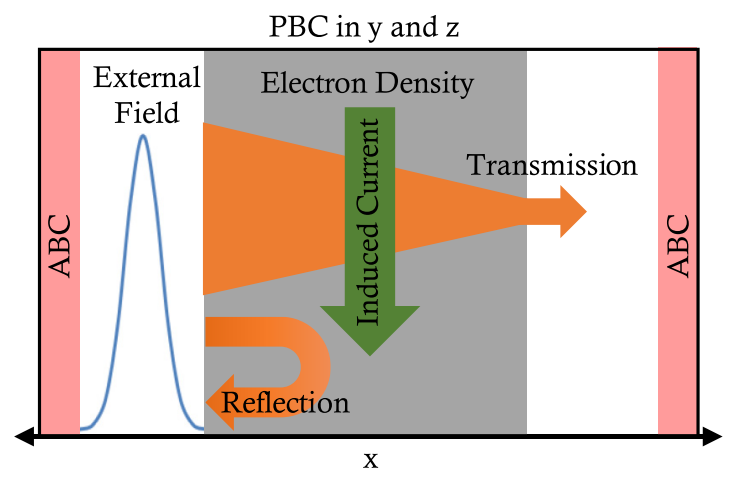

FIG. 1. Computational box containing a laser incident on the surface of a conducting layer. The laser was propagated in the $x$ direction. The dimensions of the box were $L_{x} \times L_{y} \times L_{z}$. Absorbing boundary conditions (ABCs) were added on either end of the box to absorb the EM waves. For FDTD this was a Mur-type ABC and for the RS formalism this was a CAP. PBCs were used in the $y$ and $z$ directions.

efficient multiscale approaches. For example, the propagation of the KS orbitals in the presence of atoms requires a spatial mesh with about $0.2 \AA$ and time steps around 1 attosecond. Depending on the wavelength of the EM fields, the spatial mesh representation can be much more coarse (or much finer). Due to the presence of the speed of light in the definition of $H_{\mathrm{EM}}$ in Eq. (7), the RS vector changes much faster than the KS orbitals propagated with $H_{\mathrm{KS}}$. This means that the RS propagation will require a much smaller time step than the KS propagation. This in itself is not a serious drawback since the RS propagation only advances three components of a vector and is thus a much faster calculation than the propagation of the KS orbitals.

Starting with the KS orbitals, current, and an arbitrary external vector potential at time $t=0$, time-stepping can be achieved in a straightforward manner as it is shown in the pseudo algorithm, labeled "SIMPLE" in Listing 1. First, the CAP is applied to the RS vector using Eq. (23), then the RS vector and the electron current (calculated by solving the TD-KS equation) is transformed to momentum-space using FFTs ( Listing 1, lines 2 and 3). Then Eq. (11) is evaluated in momentum-space: $\tilde{\mathbf{F}}(\mathbf{k}, t)$ is multiplied by $e^{i H_{\mathrm{EM}} \Delta t / \hbar}$ and the current term is added (Listing 1, line 4). The next steps are a Fourier transformation back to real-space and a calculation of the vector potential using Eq. (19) ( Listing 1, lines 5-8). Once the vector potential is calculated, the Taylor propagator can be used to advance the KS orbitals in time ( Listing 1, line 9). Finally, the new currents and density are calculated and the density-dependent $\mathrm{KS}$ potential $V_{\mathrm{KS}}$ is updated by solving the Poisson equation and evaluating the $V_{\mathrm{XC}}$, both using the new density. In practice, higher-order propagation schemes are preferred, such as a leapfrog algorithm which shifts the time grid between the two coupled equations by a half step. This approach does not increase the computational time significantly, yet greatly increases the accuracy of the results. The leapfrog algorithm proceeds in much the same way as the simple algorithm, but the time index is more complicated (see the algorithm in Listing 2). 
Algorithm 1 RS and KS time propagation

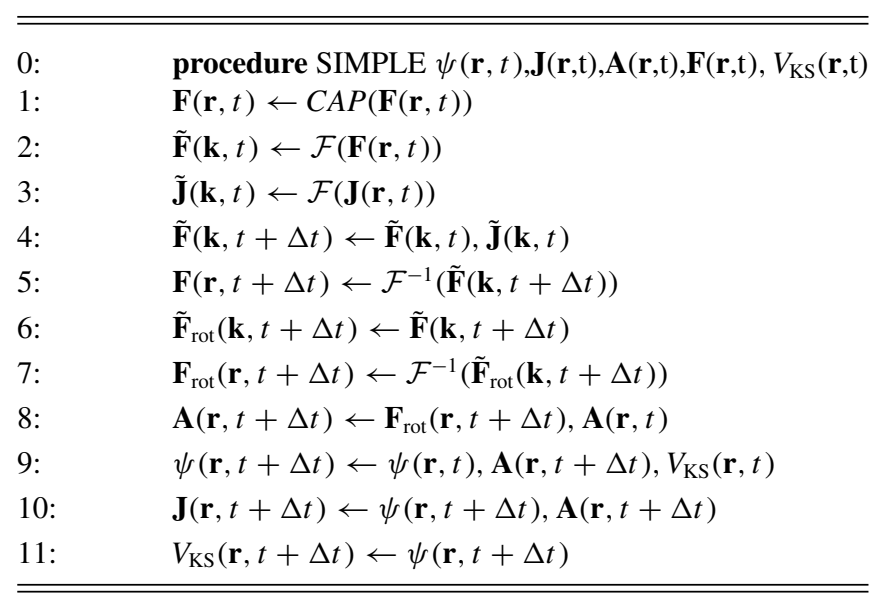

Algorithm 2 Advanced RS and KS time propagation

\begin{tabular}{ll}
$0:$ & procedure LeapFrog $\psi(\mathbf{r}, t), \mathbf{J}(\mathbf{r}, \mathrm{t}), \mathbf{A}(\mathbf{r}, t-\Delta t / 2)$, \\
& $\mathbf{F}(\mathbf{r}, t-\Delta t / 2), V_{\mathrm{KS}}(\mathbf{r}, \mathrm{t})$ \\
$1:$ & $\mathbf{F}(\mathbf{r}, t-\Delta t / 2) \leftarrow C A P(\mathbf{F}(\mathbf{r}, t-\Delta t / 2))$ \\
$2:$ & $\tilde{\mathbf{F}}(\mathbf{k}, t-\Delta t / 2) \leftarrow \mathcal{F}(\mathbf{F}(\mathbf{r}, t-\Delta t / 2))$ \\
$3:$ & $\tilde{\mathbf{J}}(\mathbf{k}, t) \leftarrow \mathcal{F}(\mathbf{J}(\mathbf{r}, t))$ \\
$4:$ & $\tilde{\mathbf{F}}(\mathbf{k}, t+\Delta t / 2) \leftarrow \tilde{\mathbf{F}}(\mathbf{k}, t-\Delta t / 2), \tilde{\mathbf{J}}(\mathbf{k}, t)$ \\
$5:$ & $\mathbf{F}(\mathbf{r}, t+\Delta t / 2) \leftarrow \mathcal{F}^{-1}(\tilde{\mathbf{F}}(\mathbf{k}, t+\Delta t / 2))$ \\
$6:$ & $\tilde{\mathbf{F}}_{\mathrm{rot}}(\mathbf{k}, t+\Delta t / 2) \leftarrow \tilde{\mathbf{F}}(\mathbf{k}, t+\Delta t / 2)$ \\
$7:$ & $\mathbf{F}_{\mathrm{rot}}(\mathbf{r}, t+\Delta t / 2) \leftarrow \mathcal{F}-1\left(\tilde{\mathbf{F}}_{\mathrm{rot}}(\mathbf{k}, t+\Delta t / 2)\right)$ \\
$8:$ & $\mathbf{A}(\mathbf{r}, t+\Delta t) \leftarrow \mathbf{F}_{\mathrm{rot}}(\mathbf{r}, t+\Delta t / 2), \mathbf{A}(\mathbf{r}, t)$ \\
$9:$ & $\mathbf{A}(\mathbf{r}, t+\Delta t / 2) \leftarrow \mathbf{A}(\mathbf{r}, t+\Delta t), \mathbf{A}(\mathbf{r}, t)$ \\
$10:$ & $\psi(\mathbf{r}, t+\Delta t) \leftarrow \psi(\mathbf{r}, t), \mathbf{A}(\mathbf{r}, t+\Delta t / 2), V_{\mathrm{KS}}(\mathbf{r}, t)$ \\
$11:$ & $\mathbf{J}(\mathbf{r}, t+\Delta t) \leftarrow \psi(\mathbf{r}, t+\Delta t), \mathbf{A}(\mathbf{r}, t+\Delta t)$ \\
$12:$ & $V_{\mathrm{KS}}(\mathbf{r}, t+\Delta t) \leftarrow \psi(\mathbf{r}, t+\Delta t)$ \\
\hline \hline
\end{tabular}

\section{F. Implementation}

The RS formalism was implemented in a real-space, realtime TDDFT code that incorporates a homogeneous background potential with a charge density defined using the Wigner-Seitz radius for a given material (e.g., lithium or aluminum). The electronic wave function is represented on a real-space grid of equal spacing in all directions. For the electrons, the application of differential operators for the first and second derivatives are evaluated using fourth-order finitedifference formulas. The velocity gauge Hamiltonian is also applied in real-space, which allows the first and second derivatives to be calculated at the same time, since the same values are needed for both. This representation significantly saves on memory bandwidth and computational time. The accuracy and efficiency of our real-space and real-time TDDFT code have been tested in many calculations [38,76,77,83].

An external field will be added to induce currents in the jellium system. The external field used in these simulation takes the form of a Gaussian laser pulse given as

$$
E_{z}(x, t)=E_{0} e^{-\left(t-t_{0}-x / c\right)^{2} / \alpha^{2}} \sin (\omega(t-x / c)) \Gamma(x, t) .
$$

Here $t_{0}$ is the pulse shift, $\alpha$ controls the width of the pulse, $\omega$ gives the frequency, and $c$ is the speed of light. $\Gamma$ is a ramping
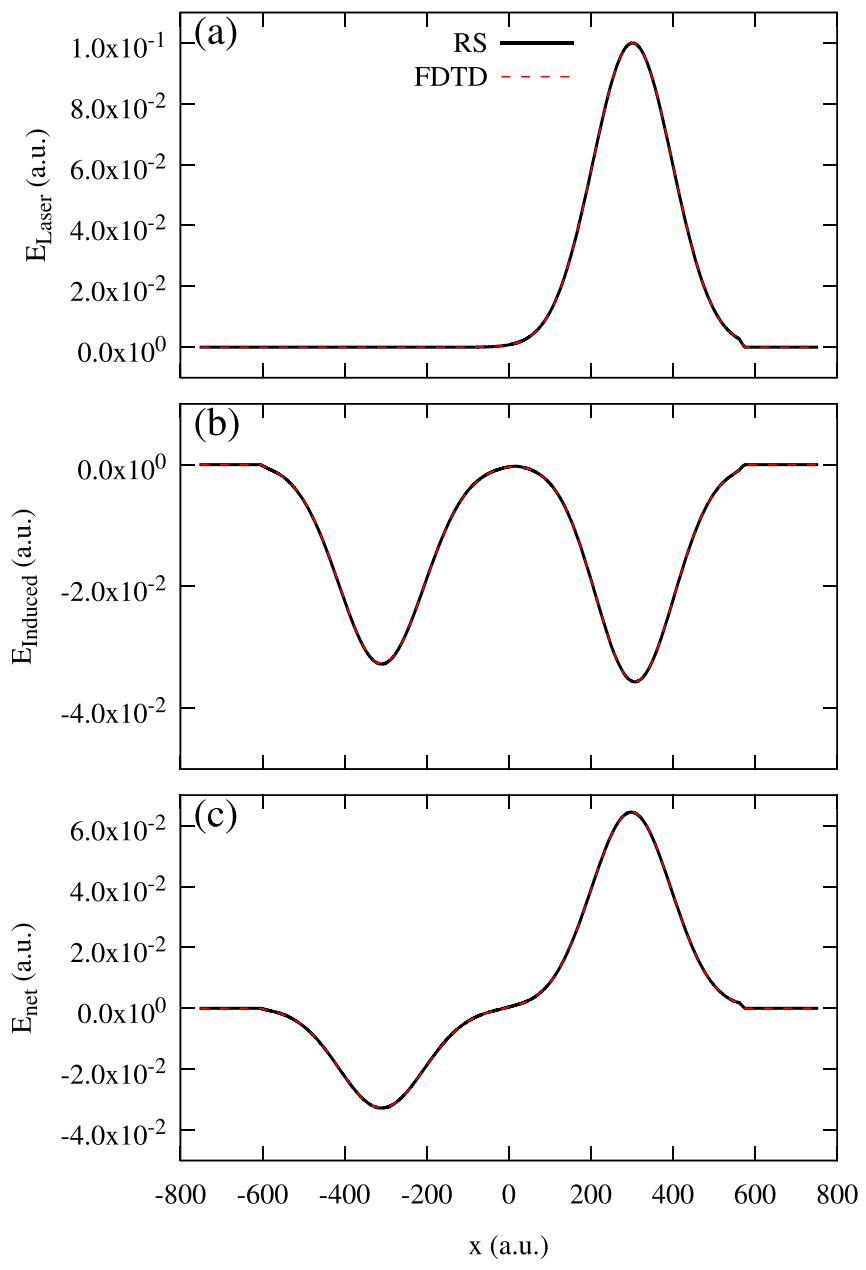

FIG. 2. A comparison between the FDTD and RS methods at $t=4.2$ a.u. for a laser pulse incident upon a uniform conducting sheet. For the RS method $\Delta t_{\mathrm{EM}}=0.0015$ and for the FDTD method $\Delta t_{\mathrm{FDTD}}=0.00075$. The parameters of the computational box were $L_{x}=1500, L_{y}=L_{z}=20$ a.u. with grid spacings of $\Delta x=\Delta y=$ $\Delta z=0.5$ a.u.. The laser parameters were $E_{0}=0.1$ a.u., $t_{0}=2$ a.u., $\alpha=1$ a.u., and $\omega=0$. The sheet was positioned in the middle of the cell perpendicular to the $x$-axis. The sheet was 60 a.u. wide, and its conductivity was set to be $\sigma=0.1$ a.u. CAPs were used in the $x$ direction and PBCs were used in the $y$ and $z$ directions. Note, the two curves are on top of each other.

function given as

$$
\Gamma(x, t)= \begin{cases}(t-x / c) / t_{\gamma}, & \text { if } t-x / c<=t_{\gamma} \\ 1, & \text { if } t-x / c>t_{\gamma},\end{cases}
$$

which is used to ensure that the field within the jellium is zero at the beginning of the simulation. The parameter $t_{\gamma}$ is the ramping time which is set to 0.1 a.u. unless otherwise noted. The parameters used in the calculations are equal or similar to the parameters of typical FDTD simulations presented in Ref. [16] and their concrete values will be specified in the figure captions.

In the jellium model the core electrons and the nuclei are modeled as the uniform positive background and the valence electrons are treated explicitly. In the examples the jellium is defined by the valence electron density, the number of 

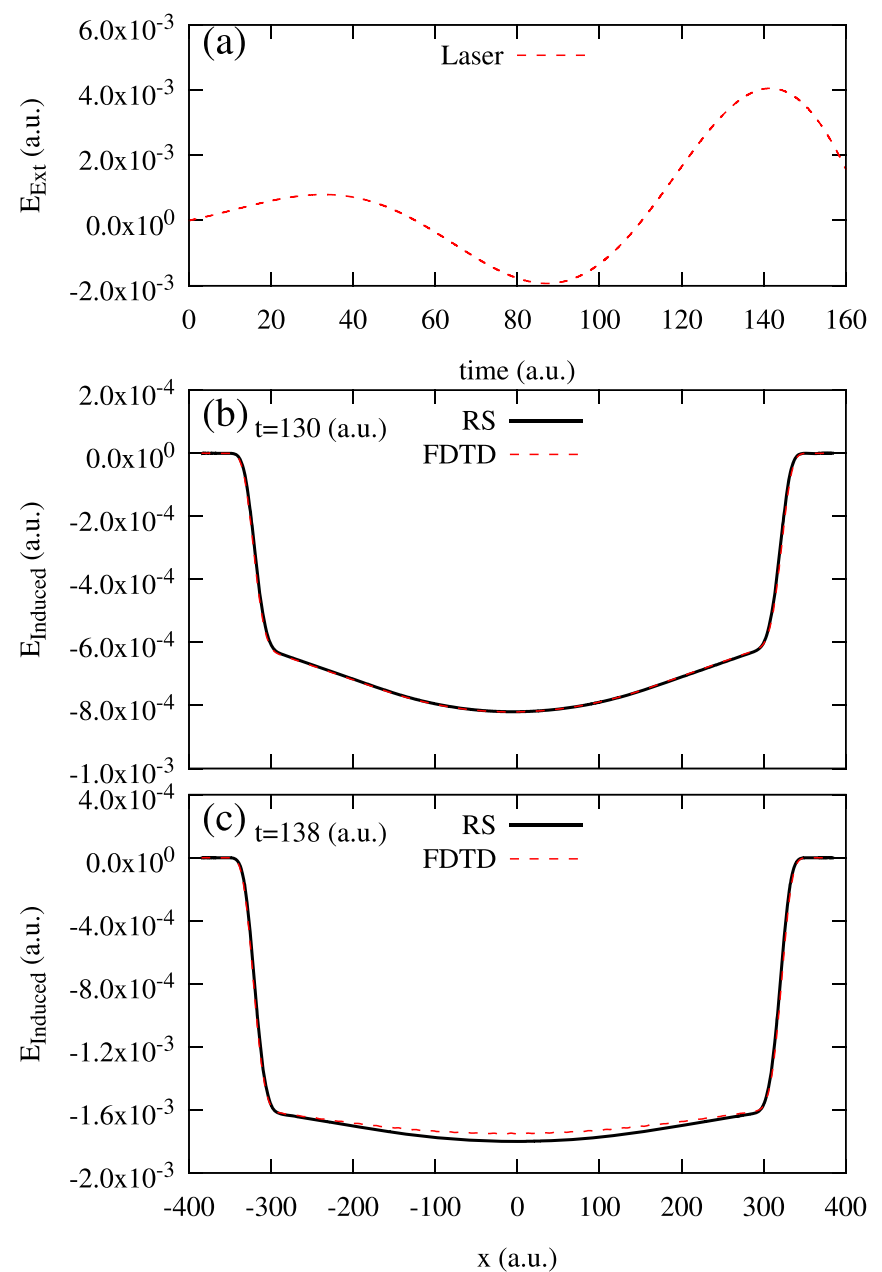

FIG. 3. A comparison between FDTD and RS solutions at $t=$ 130 a.u. and $t=138$ a.u. for a laser pulse incident upon a wide layer of lithium-jellium. The time step used in the solution of the TD-KS equations was $\delta t=0.02$ a.u. For the RS method, $\Delta t=0.02 / 20$ a.u., and for the FDTD method, $\Delta t=0.02 / 2000$ a.u. The simulation cell size used was $L_{x}=767.5$ a.u., $L_{y}=L_{z}=3.5$ a.u., and the grid spacings were $\Delta x=\Delta y=\Delta z=0.5$ a.u.. The parameters of the laser pulse [see Eq. (34)] were $E_{0}=0.1$ a.u., $t_{0}=400$ a.u., $\alpha=206$ a.u., and $\omega=0.05695$ a.u. (which corresponds to $800 \mathrm{~nm}$ wavelength). The width of layer was 328 a.u., containing 36 electrons. CAPs were used in the $x$ direction and PBCs were used in the $y$ and $z$ directions. The Top panel shows the incident electric field in time. The middle and bottom panels show the average electric field along the $x$ axis at different times. Note, in the middle panel the two curves are on top of each other.

electrons per volume. We will use lithium-jellium with one valence electrons and aluminum-jellium with three valence electrons interacting with the uniform positive background charge representing the ionic cores. These jellium models work remarkably well for simple metal clusters [84].

For the FDTD simulations we have used the computer code accompanied with Ref. [16]. This code implements the FDTD solution of Maxwell's curl equations over a threedimensional Cartesian space lattice. The grid is terminated by perfectly matched layers absorbing boundary conditions. We have tested the code using numerical examples of Ref. [16].
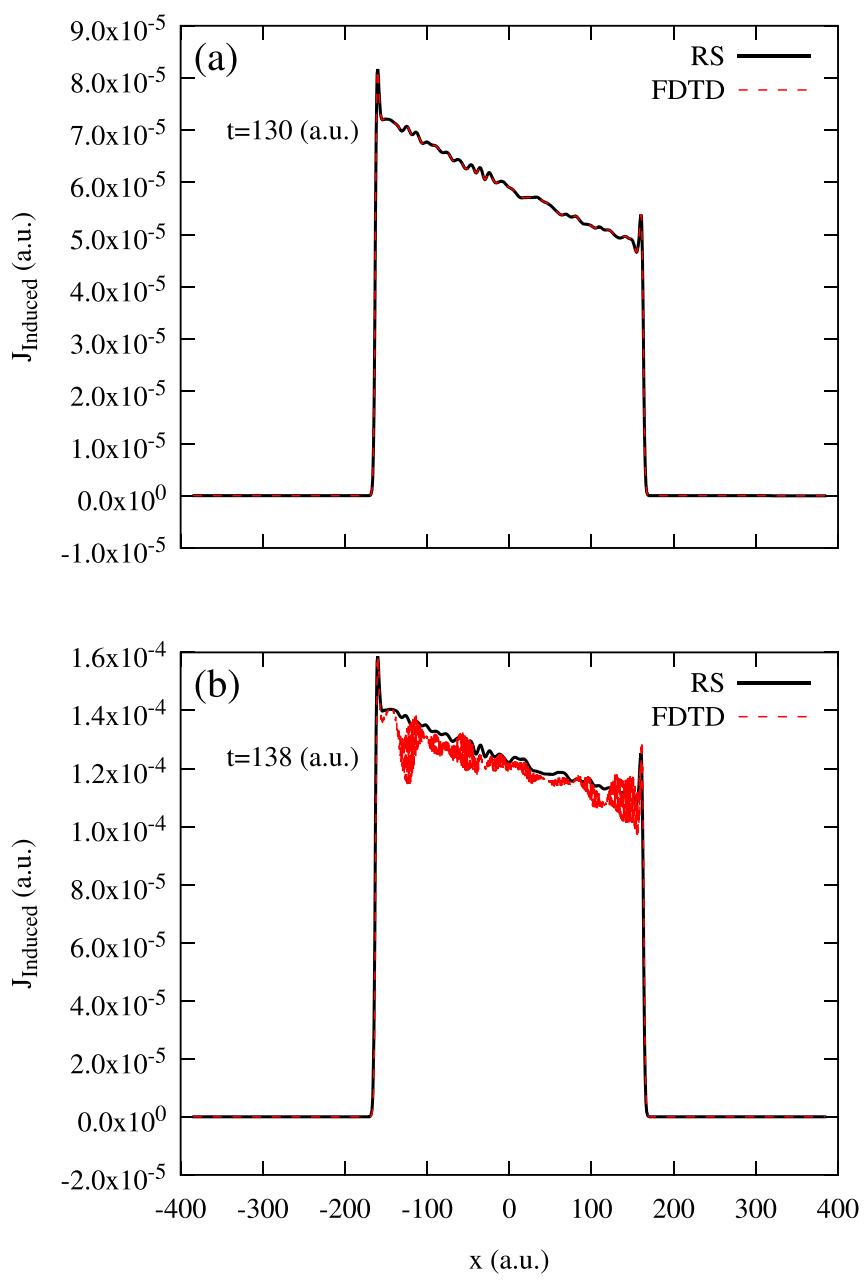

FIG. 4. A comparison of the current calculated by the FDTD and RS methods corresponding to the same system and times as those shown in Fig. 3. Note, for the top panel the two curves are on top of each other.

\section{RESULTS AND DISCUSSION}

\section{A. Comparison of FDTD and RS methods: A conducting layer}

The input of the RS and FDTD approach is the external field and the electric current. First we compare the FDTD and RS approaches in a macroscopic case for a uniform conducting layer. This problem is macroscopic in the sense that we rely on macroscopic material properties-that is we use Ohm's law, $J(\mathbf{r})=\sigma(\mathbf{r}) E(\mathbf{r})$, to get the electric current. In a "microscopic" simulation of the coupled Maxwell-TDDFT system, the electric currents would be calculated by TDDFT.

To compare FDTD and RS on the same footing, we use the same simulation box and grid spacing. A sketch of the system is shown in Fig. 1. A laser pulse incident upon a uniform conducting sheet will be propagated. The electric field inside the sheet is expected to induce a current according to Ohm's law. This current, as a material response, will be used to update the EM fields via Maxwell's equations. We note that no modification to the permittivity of free space is used. Throughout this paper, atomic units (a.u.) have been used. 


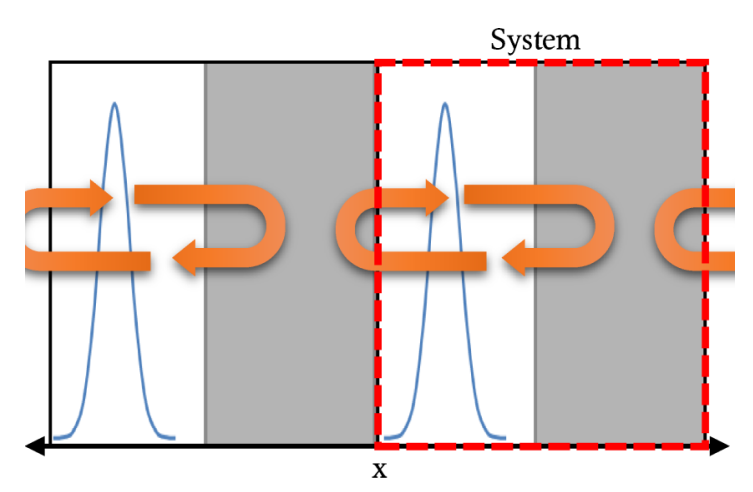

FIG. 5. System setup for a rigorous test of time propagation algorithms with the RS method. Initially a very short laser pulse is to be added to the box. The pulse then is to reflect off of the left and right sides of the box, going through the periodic boundary in the $x$ direction.

The results are given in Fig. 2, which shows the external laser field (labeled $E_{\text {Laser }}$ ), the field due to the response of the material (labeled $E_{\text {induced }}$ ), and the sum of the laser and material (labeled $E_{\text {net }}$ ). The induced current field in the conducting sphere produces an electric field that is counter to the external field. This will propagate outward in both directions. Due to the principle of linear superposition, the induced pulse cancels out some of the incident pulse, resulting in the observed effect of partial reflection of the incident pulse.

The RS method propagates the EM fields just as well as the FDTD method. The time step taken with the RS method can be larger by a factor of 3 , due to the higher accuracy of the spatial derivatives. The time step and accuracy is also better in the RS approach because it uses a higher-order solution of the differential equations then the first-order FDTD. This example shows that the RS approach can be used as a Maxwell solver. The FDTD method is very fast and efficient, and in problems based on material properties it would normally be preferred to using the RS formalism.

\section{B. Comparison of FDTD and RS method: Real electrons}

In this example we do a similar calculation as in the previous case but with real electrons; the material response will be calculated by TDDFT. We test both the RS and the FDTD methods in coupling the Maxwell and the KS equations. The input of the RS and FDTD step is the electric current (calculated by TDDFT) and the external EM field. The output is the vector potential that will be used in TDDFT.

In this example we have the same computational setup as shown in Fig. 1, but the layer is a system of a homogeneous positive background potential and electrons. When the FDTD method is applied to a system with real electron density, the stability becomes a major issue. Two snapshots of the simulation are shown in Fig. 3. At $t=130$ a.u. the FDTD and the RS methods give similar results, but soon thereafter the FDTD solution starts to diverge and eventually blows up. We have found that the FDTD time propagation fails in most cases due to its instabilities for time steps as small as even $\Delta t=10^{-5}$ a.u.. The snapshots of the current at the same times
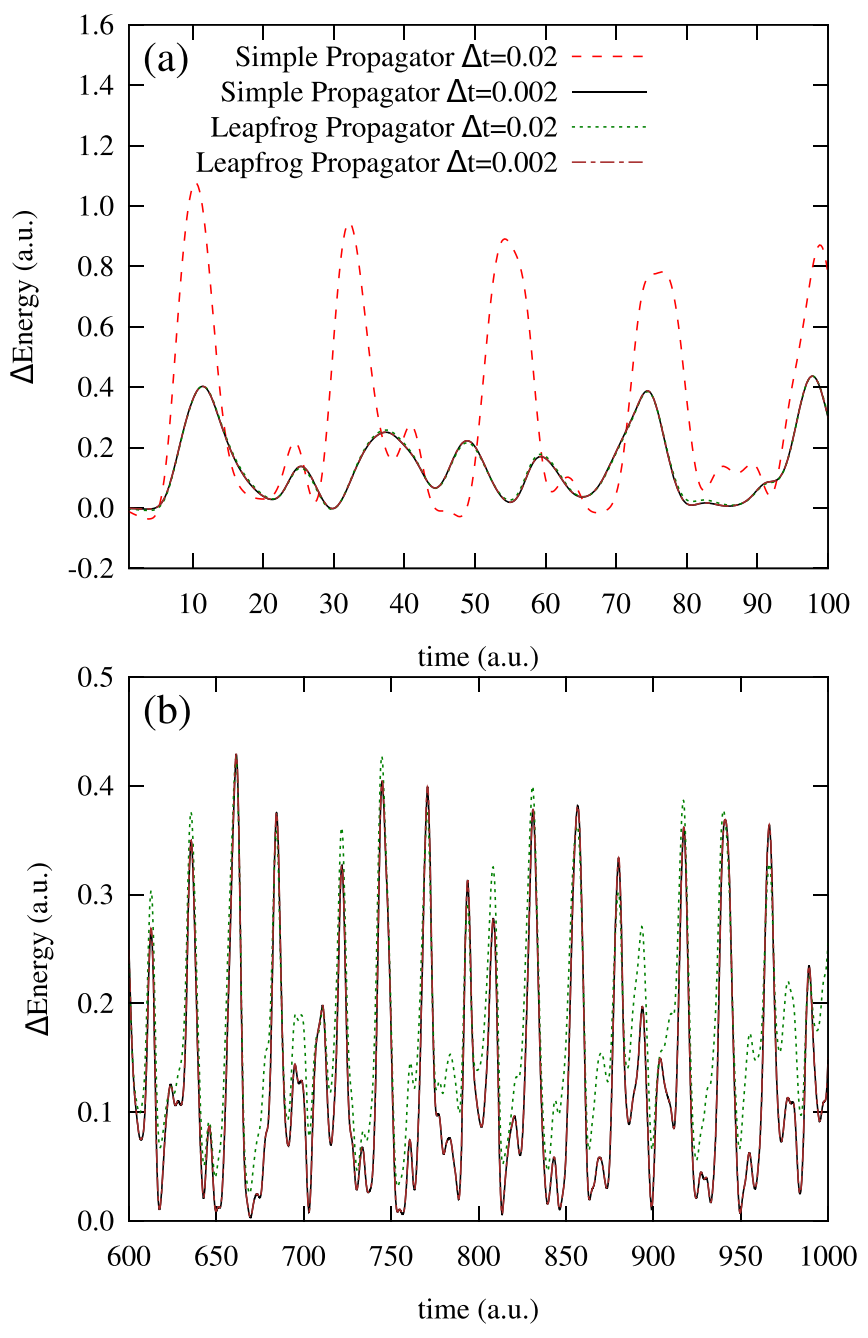

FIG. 6. The change in the electronic energy in time using the simple and leapfrog time propagation schemes using time steps of 0.02 and 0.002 a.u. for electrons with 20 RS steps per TD-KS time step. The simulation cell size was $L_{x}=2304$ a.u., $L_{y}=L_{z}=3$ a.u. with grid spacings of $\Delta x=\Delta y=\Delta z=0.5$ a.u.. The parameters of the laser pulse [see Eq. (34)] were $E_{0}=0.1$ a.u., $t_{0}=0$ a.u., $\alpha=2$ a.u., and $\omega=0$. PBCs were used in each direction. The width of the jellium, corresponding to the gray area in Fig. 5, was 777 Bohr. The curves for the simple propagator and the leapfrog propagator are on top of each other for the 0.002 a.u. time step.

are shown shown in Fig. 4. The current becomes very noisy in FDTD which then leads to divergence.

One possible reason for the divergence of the FDTD solution is that only first-order spatial derivative approximations are used. Therefore, there will be some error associated with each field at each grid point. Because all of the quantities are coupled, numerical instabilities can lead to catastrophic failure of the time propagation. Another issue may be the divergence of the vector potential. The velocity gauge Hamiltonian is valid only for vector potentials in the Coulomb gauge, for which $\boldsymbol{\nabla} \cdot \mathbf{A}=0$. We have performed HDs on FDTD obtained electric field both using FD operators and the Fourier components (the same way as the RS vector), and in both the same instabilities arise so that the electron density always diverges. It is surmised that if more accurate 


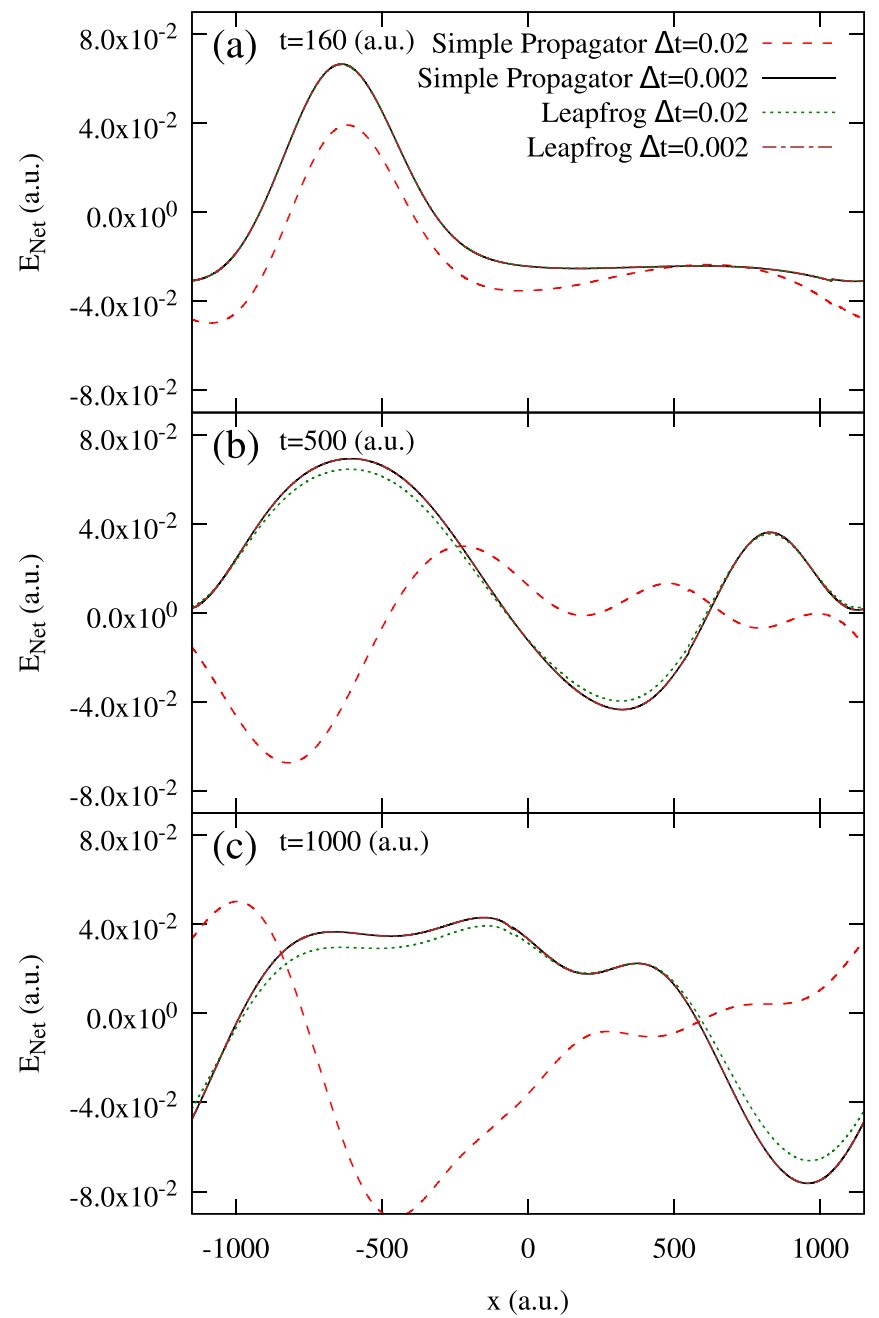

FIG. 7. Electric field vector at given times during the rigorous time-step test simulation using the simple and leapfrog time propagation schemes with time-step sizes of 0.02 and 0.002 a.u. for electrons and 20 RS steps per TD-KS time step.

derivatives were used with the FDTD method, the instabilities would be less, and time propagation would be possible. However, such increased order finite-difference formulas would dramatically reduce the speed and efficiency of the FDTD approach.

\section{RS propagation rigorous time-step test}

To more rigorously test the accuracy of the RS time propagation scheme, the dynamics of the test system must be driven by the induced fields, and not some external field. If an extended exciting pulse were used, the induced fields would be absorbed by the CAPs before they could significantly excite other electrons and induce counter currents. One way to achieve a long simulation in which induced fields have a continued influence is to remove the CAPs and use a very short excitation pulse. With the CAPs removed the system is periodic in all directions. Then if a thick layer of conductive material is used, the short pulse will be partially reflected and approach from the opposite side, as shown in Fig. 5.
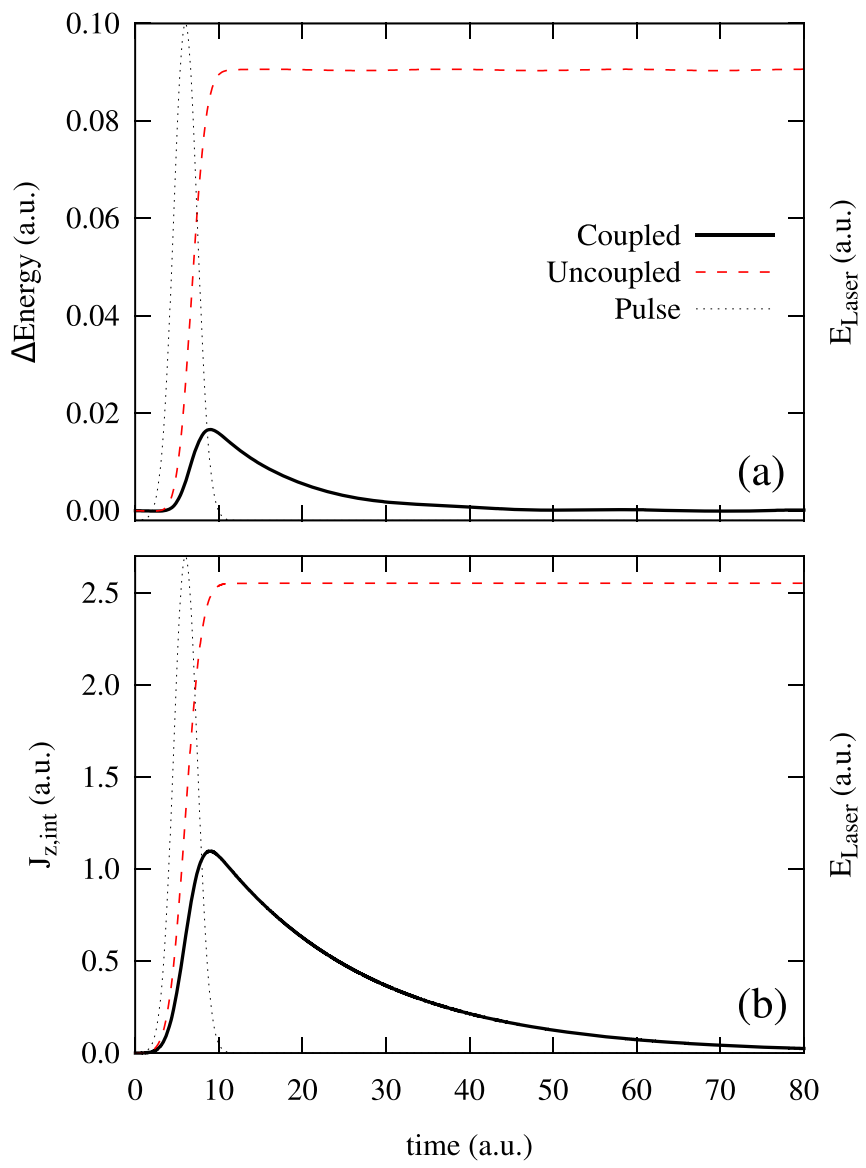

FIG. 8. Comparison of a simulation with and without the coupling of the induced current, showing the change in electronic energy (top) and the electron current (bottom). The system was lithiumjellium consisting of 36 electrons in a 328 a.u. wide sheet contained within a box of $L_{x}=576$ a.u., $L_{y}=L_{z}=4$. a.u. with grid spacings of $\Delta_{x}=\Delta_{y}=\Delta_{z}=0.5$ a.u. CAPs were used in the $x$ direction and PBCs were used in the $y$ and $z$ directions. The system was excited by a Gaussian pulse with peak electric field of $E_{0}=0.02$ a.u., a width of $\alpha=2.0$ a.u. and a shift of $t_{0}=6$ a.u. It is seen that without the coupling of the Maxwell and KS equations, the system is put into an excited state which does not relax to the ground state.

These PBCs were applied to a system of lithium-jellium in a long-narrow box with different time steps and using both integration methods, the results of which are shown in Fig. 6.

The leapfrog integrator appears to be well-converged for time-step sizes as large as even $\Delta t=0.02$ a.u., seeing as the solution using these time steps closely agrees with that of $\Delta t=0.002$ a.u.. This is not the case, however, for the simple integrator which performs poorly at such larger timestep sizes. In each of these simulations, $20 \mathrm{RS}$ time steps were used per time step of the KS equations. Since 0.02 a.u. is practically the longest time step possible for propagating the TD-KS equations on a grid of this spacing, the leapfrog integrator is the obvious choice. There is some deviation in the energies at later times in the simulation; however, there is some dependency on the time step for the time propagation of the KS equations alone, since they are nonlinear. The resulting total electric field is well matched between 0.02 and 0.002 a.u. time steps, as shown in Fig. 7 at various time points. 


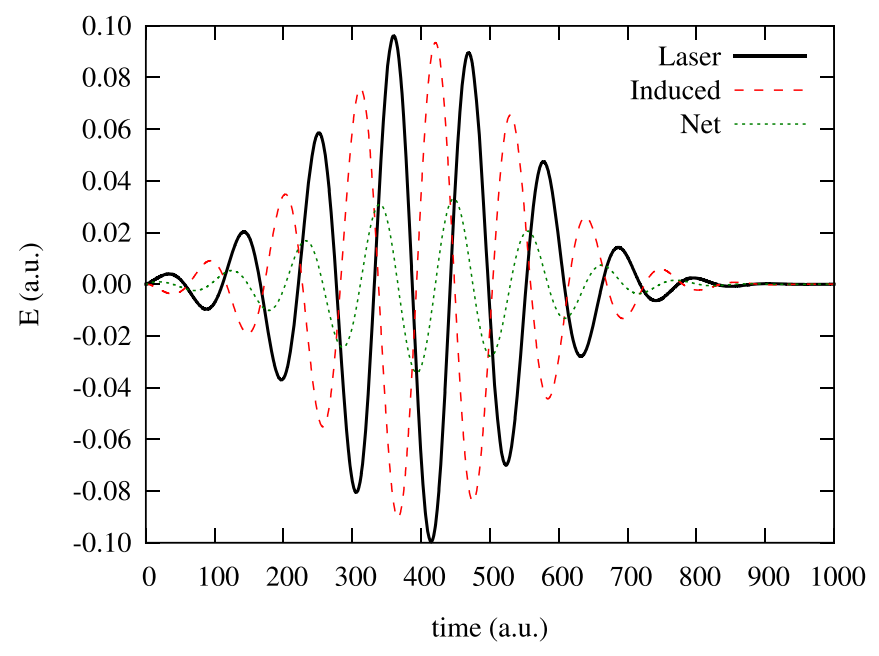

FIG. 9. Comparison of the transmitted electric field (taken at the beginning of the right CAP) resulting from a strong laser pulse incident from the left on a 232 a.u. thick sheet of aluminum-jellium of 400 electrons within a box of $L_{x}=320$ a.u., $L_{y}=L_{z}=8$. a.u. with grid spacings of $\Delta_{x}=\Delta_{y}=\Delta_{z}=0.5$ a.u. CAPs were used in the $x$ direction and PBCs were used in the $y$ and $z$ directions. The system was excited by a Gaussian pulse with a peak electric field of $E_{0}=0.1$ a.u., a width of $w=206$ a.u., $\omega=0.05695$ a.u., and a shift of $t_{0}=400$ a.u.. A ramp time of $\gamma=0.5$ a.u. was used for this simulation.

These test calculations show that the additional coupling to the Maxwell equations does not put any restriction on the maximum time step of the KS equations. The accuracy of the leapfrog time integration scheme with a time step of 0.02 a.u for the KS equations and $0.02 / 20=0.001$ a.u. for the RS equations, is quite good, and is used for all calculations throughout the rest of the paper. Higher-order means of time propagation such as the predictor corrector or Runge-Kutta methods exist; however, they will require more applications of the exponential operators and FFTs per step.

\section{Comparison to uncoupled case}

In this test case we will compare two calculations: one in which in the KS equations are coupled to the Maxwell equations and one in which there is no such coupling. In the uncoupled case any incident EM waves propagates through space as if there were no electrons present, that is $\mathbf{J}=0$ is plugged into Eq. 14. A simulation was performed using a very short pulse to place a jellium sheet into an excited state, using both Maxwell-coupled and Maxwell-uncoupled time propagations. In the Maxwell-uncoupled system, the pulse excites the electrons and causes significant electron current, as shown in Fig. 8. After the pulse has passed through the medium, the system is still in an excited state, and, since there is no resistance in this simulation (no scattering by phonons), the current continues. In the Maxwell-coupled system the pulse excites electrons and the electron current generates an electric field that de-excites the electrons. The resulting energy decays back to the ground state and the current decays back to zero. This shows that the Maxwell-coupled system has the proper behavior for conductive materials.
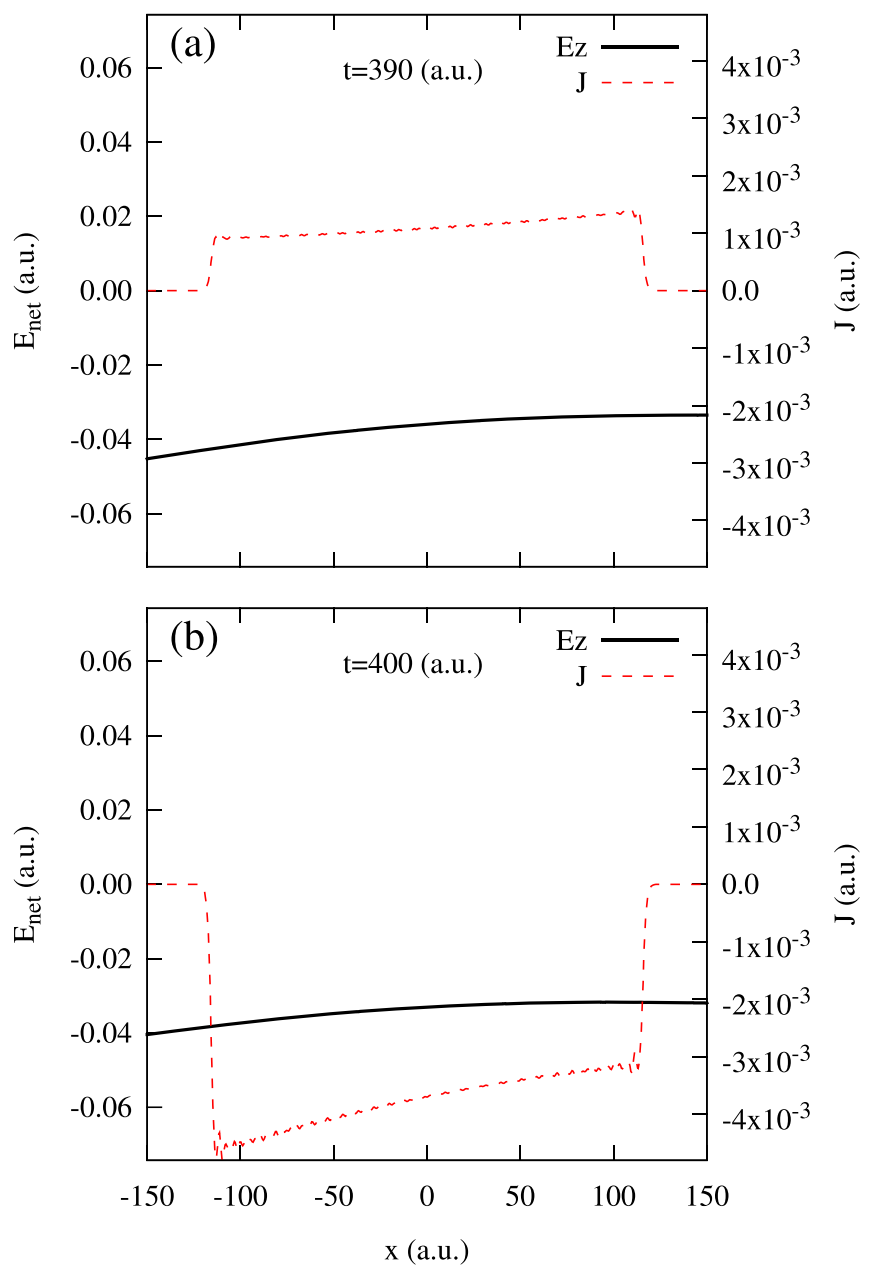

FIG. 10. Comparison of the net electric field and current at given time points resulting from a strong laser pulse incident from the left on a sheet of aluminum-jellium. The system parameters are given in Fig. 9

\section{E. Longer exciting pulses}

Using longer pulses, the frequency of the excitation is closer to the energy of the electrons, so they more completely respond to the external field. By using thick jellium sheets, the incident pulse may be nearly completely reflected or modulated. As the light penetrates the medium, the intensity will diminish and create a broad range of pulse intensities. This has applications in the calculation of harmonic spectra.

The results of a simulation with a sheet of aluminumjellium irradiated by a strong laser pulse are shown in Fig. 9 . The electric field is not uniform as the external electric field penetrates the sheet since the field induces currents stronger on the side of incidence. This is shown in Fig. 10. The nonuniformity of the electric field from a laser pulse as a function of depth is modeled by this approach.

\section{F. Simulation of jellium spheres}

Next we test the approach using a jellium model of quantum dots. A simulation was performed on an aluminumjellium sphere of radius 12.8 a.u., corresponding to a diameter 


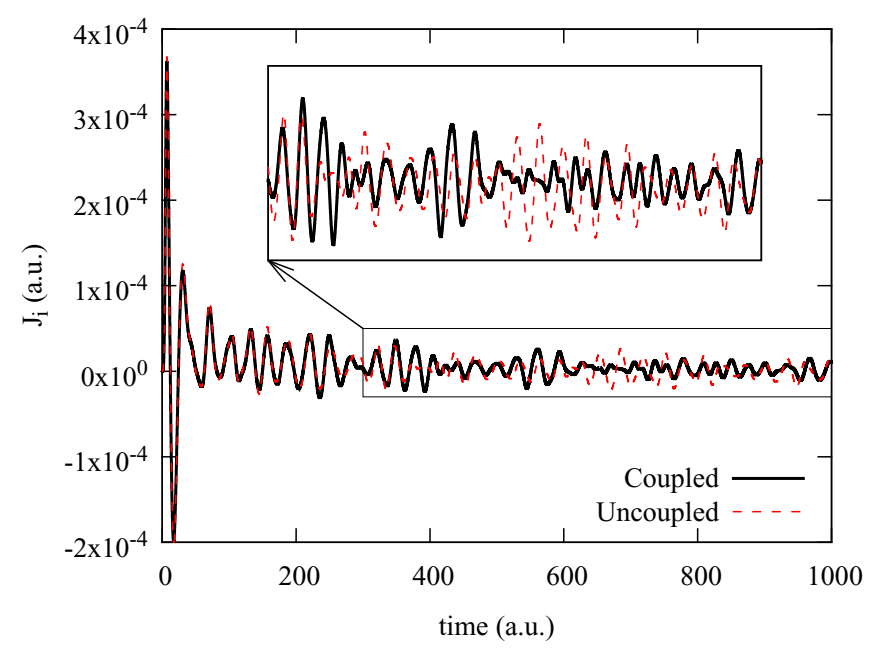

FIG. 11. Comparison of the cell average currents in time resulting from a strong laser pulse incident from the left on a sphere of aluminum-jellium of 236 electrons in a box of $L_{x}=96$ a.u., $L_{y}=L_{z}=30$. a.u. with grid spacings of $\Delta_{x}=\Delta_{y}=\Delta_{z}=0.5$ a.u. CAPs were used in the $x$ direction and PBCs were used in the $y$ and $z$. directions. The system was excited by a Gaussian pulse with a peak electric field of $E_{0}=0.1$ a.u. with a width of $\alpha=2$ a.u. and a shift of $t_{0}=5$ a.u.. The inset more clearly shows the differences in current as the simulation progresses.

of $1.4 \mathrm{~nm}$, which is very small in terms of actual nanoparticles. This small sphere nevertheless contains 236 electrons. A comparison of the electron currents with and without coupling the TD-KS and the Maxwell equations is shown in Fig. 11. The effect of simulating the dynamics with the Maxwell equations is small early in the simulation, but differences do appear at later times. This simulation shows how the electron induced currents for systems as small as a single nanoparticle can influence the electron dynamics.

\section{G. Propagation in inhomogeneous system}

In the previous test cases the medium in which the EM waves were propagated was homogeneous, except for the abrupt surfaces. The electron density is very inhomogeneous in atomistic systems. We have two examples to mimic the effect of nonlocality: a system with periodically repeated local potentials and a system of periodically repeated tilted potential wells.

In the case of periodically repeated local potentials, we use a wedge shape, as seen in Fig. 12. We have used a wedge rather then a sphere to mimic the effect of bonds which are directed into a certain direction. The resulting net electric field is seen to be strongly modulated by the wedge shape, as seen in Fig. 12.

The second example with a tilted potential well models a medium that contains certain lattice planes that are far more conductive in certain directions, as is the case with graphene. A simulation of such a situation using aluminum-jellium sheets is shown in Fig. 13. Here, as it is expected, the current will be different in different directions.
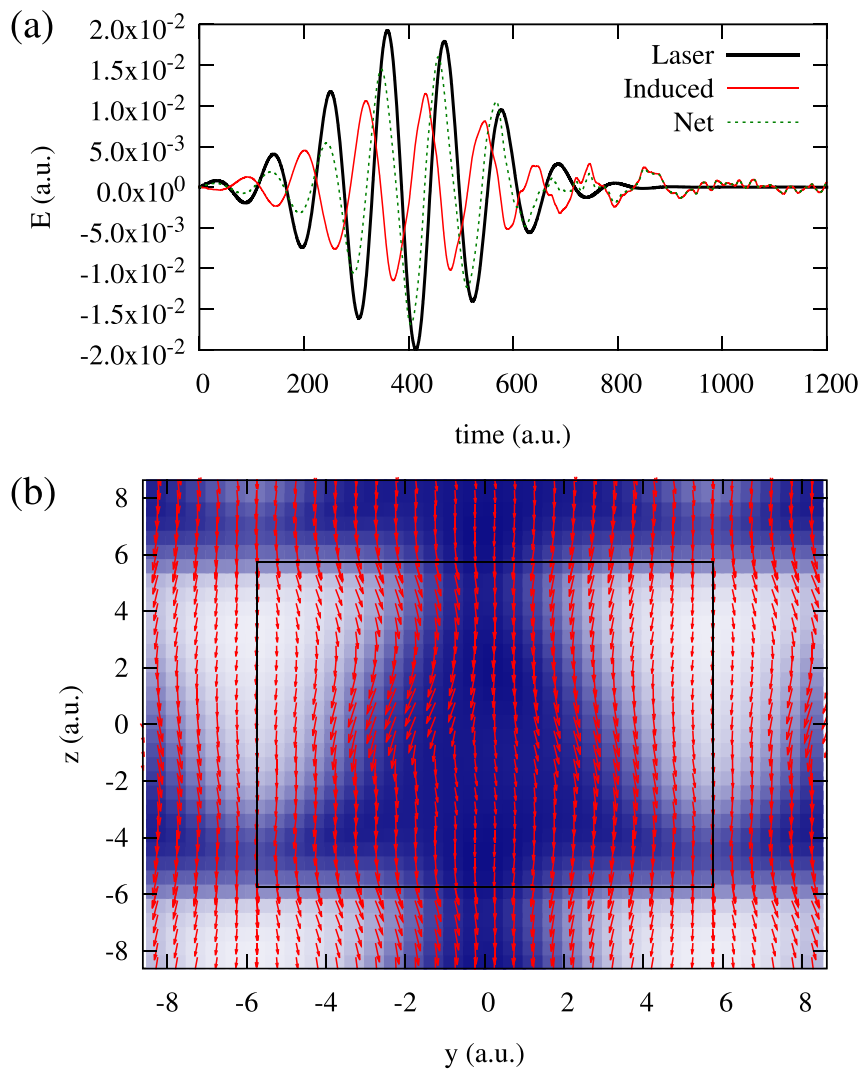

FIG. 12. Vector plot of the current in the $y-z$ plane resulting from a strong laser pulse incident from the left on a repeating wedge of aluminum-jellium 100 Bohr thick containing 258 electrons (top) and the electric field in time (bottom). The dark color shows the region where the electron density is large. Each wedge was contained within a box of of $L_{x}=160$ a.u., $L_{y}=L_{z}=12$ a.u. with grid spacings of $\Delta_{x}=\Delta_{y}=\Delta_{z}=0.5$ a.u. CAPs were used in the $x$ direction and PBCs were used in the $y$ and $z$ directions. The images have been wrapped to illustrate the periodicity and the periodic boundaries are shown as a box. The system is excited by a Gaussian pulse with peak electric field of $E_{0}=0.02$ a.u. with a width of $\alpha=206$ a.u., a shift of $t_{0}=400$ a.u., and $\omega=0.05695$ a.u.

\section{SUMMARY}

We have implemented and tested the Riemann-Silberstein vector formalism in momentum-space. This approach has been first proposed and implemented in Ref. [61] in realspace. Both approaches have advantages and disadvantages, and which is better suited depends on the concrete physical system being simulated.

One of the main advantages of the momentum-space formalism is that the EM Hamiltonian is diagonal and the time propagation with FFTs is simple, efficient and accurate. Another benefit is the straightforward use of the Helmholtz decomposition for the extraction of the desired vector potential. The easy implementation within time-dependent plane wave TDDFT codes (see, e.g., the Volkov basis approach in Ref. [76]) is also an advantage.

We have compared the RS and FDTD solution of the Maxwell equations to show the accuracy of the RS approach. We have found that it is difficult to couple the FDTD approach 
(a)
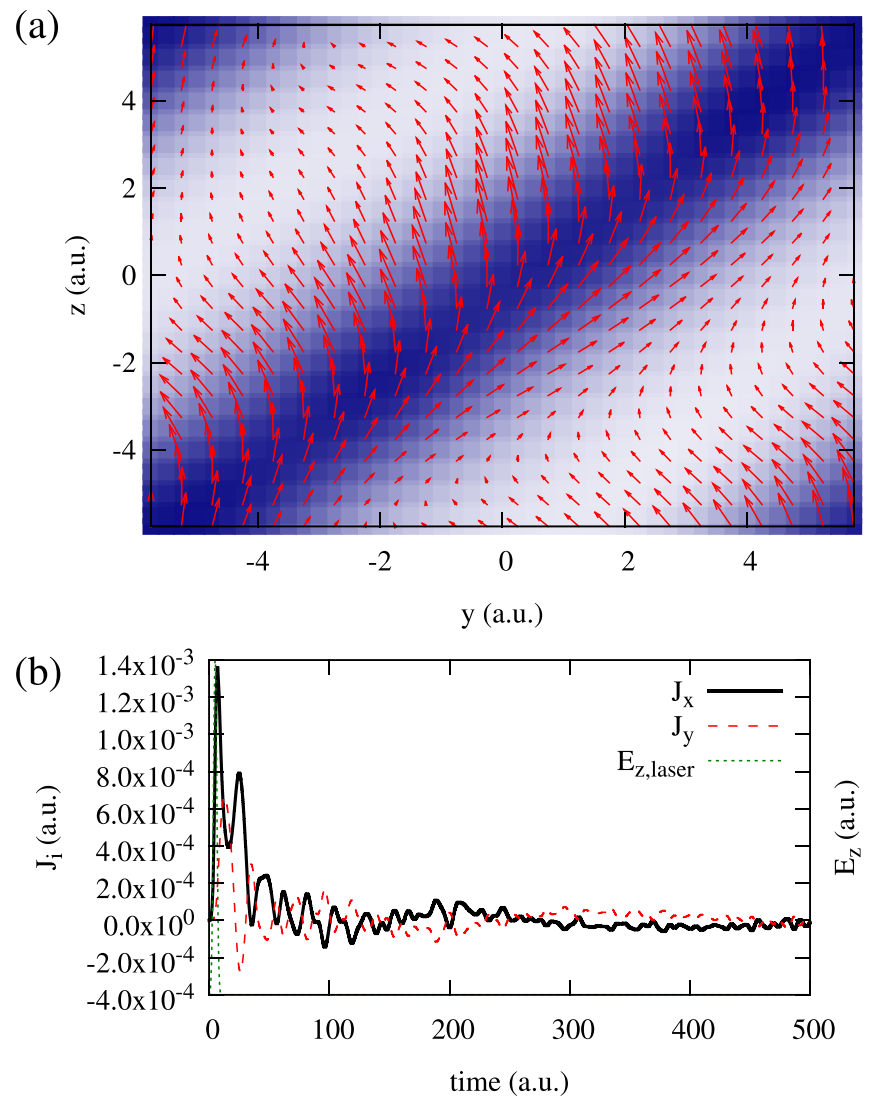

FIG. 13. Vector plot of the current in the $y-z$ plane (top) and the electric field (bottom) resulting from a strong laser pulse incident from the left on a repeating slit of aluminum-jellium 108 Bohr thick containing 200 electrons that has been rotated at 45 degrees. The dark color shows the region where the electron density is large. The sheet was contained within a box of $L_{x}=256$ a.u., $L_{y}=L_{z}=12$ a.u. with grid spacings of $\Delta_{x}=\Delta_{y}=\Delta_{z}=0.5$ a.u. CAPs were used in the $x$ direction and PBCs were used in the $y$ and $z$ directions. The system is excited by a Gaussian pulse with peak electric field of $E_{0}=0.1$ a.u. with a width of $\alpha=2$ a.u. and a shift of $t_{0}=5$ a.u. due to the TDDFT solver due to the different time scales and to the inaccuracy of the first-order finite-difference representation used in FDTD. This problem does not persist if Maxwell's equations are solved in Riemann-Silberstein representation with a finite-difference discretization in real-space, since then higher-order discretizations can be easily used [61].

The momentum-space representation invokes periodic boundary conditions which would limit the applicability of the approach. Motivated by the complex absorbing potentials used in quantum mechanics, we have designed and optimized a complex absorbing potential for the EM Hamiltonian. The CAP is in real-space and it efficiently diminishes the transmission and reflection of EM waves at the boundary.

A leapfrog algorithm was implemented and tested for coupled propagation of the Maxwell and TDDFT equations. It exhibited substantially increased accuracy compared to the simple time propagation which only uses the EM fields and electron wave functions in a single point in time. Various further development is possible and needed to make the approach multiscale in time and space.

The approach has been tested on various systems, including jellium sheets, a jellium sphere, and periodically repeated confining potentials. The next step is obviously the inclusion of atoms using ionic potentials. As the TDDFT code used in these calculations already has the capability to describe atomic systems $[38,54,55,76]$, this step does not seem to be a problem, but it requires careful testing. This is left for future work.

The coupled Maxwell TDDFT approach enables description of the microscopic EM fields in quantum mechanical simulations. Using this approach one does not have to rely on approximations to extract enhanced fields or absorption spectra using material properties. The simulated emitted EM fields give direct access to observables. The coupled Maxwell TDDFT simulation has important potential applications in nano-optics, nanoplasmonics, and other physical applications where the strong light matter interaction plays important role.
[1] N. Yu, P. Genevet, M. A. Kats, F. Aieta, J.-P. Tetienne, F. Capasso, and Z. Gaburro, Science 334, 333 (2011).

[2] A. Boltasseva and H. A. Atwater, Science 331, 290 (2011).

[3] P. J. Schuck, D. P. Fromm, A. Sundaramurthy, G. S. Kino, and W. E. Moerner, Phys. Rev. Lett. 94, 017402 (2005).

[4] A. G. Curto, G. Volpe, T. H. Taminiau, M. P. Kreuzer, R. Quidant, and N. F. van Hulst, Science 329, 930 (2010).

[5] J. N. Anker, W. P. Hall, O. Lyandres, N. C. Shah, J. Zhao, and R. P. Van Duyne, Nat. Mater. 7, 442 (2008).

[6] H. Xu, E. J. Bjerneld, M. Käll, and L. Börjesson, Phys. Rev. Lett. 83, 4357 (1999).

[7] D.-K. Lim, K.-S. Jeon, H. M. Kim, J.-M. Nam, and Y. D. Suh, Nat. Mater. 9, 60 (2010).

[8] P. Berini and I. De Leon, Nat. Photon. 6, 16 (2012).

[9] H. A. Atwater and A. Polman, Nat. Mater. 9, 205 (2010).

[10] A. Polman and H. A. Atwater, Nat. Mater. 11, 174 (2012).
[11] D. L. Jeanmaire and R. P. V. Duyne, J. Electroanal. Chem. Interfacial Electrochem. 84, 1 (1977).

[12] S. Nie and S. R. Emory, Science 275, 1102 (1997).

[13] A. Aubry, D. Y. Lei, A. I. Fernández-Domínguez, Y. Sonnefraud, S. A. Maier, and J. B. Pendry, Nano Lett. 10, 2574 (2010).

[14] J. A. Schuller, E. S. Barnard, W. Cai, Y. C. Jun, J. S. White, and M. L. Brongersma, Nat. Mater. 9, 193 (2010).

[15] A. Elsherbeni and V. Demir, The Finite Difference Time Domain Method for Electromagnetics: With MATLAB Simulations (SciTech Publishing, Raleigh, NC, 2009).

[16] A. Taflove and S. Hagness, Computational Electrodynamics: The Finite-Difference Time-Domain Method, 3rd ed. (Artech House, Norwood, MA, 2005).

[17] K. J. Savage, M. M. Hawkeye, R. Esteban, and A. G. Borisov, Nature 491, 574 (2012).

[18] J. A. Scholl, A. L. Koh, and J. A. Dionne, Nature 483, 421 (2012). 
[19] C. Ciracì, R. T. Hill, J. J. Mock, Y. Urzhumov, A. I. FernándezDomínguez, S. A. Maier, J. B. Pendry, A. Chilkoti, and D. R. Smith, Science 337, 1072 (2012).

[20] S. F. Tan, L. Wu, J. K. Yang, P. Bai, M. Bosman, and C. A. Nijhuis, Science 343, 1496 (2014).

[21] H. Duan, A. I. Fernández-Domínguez, M. Bosman, S. A. Maier, and J. K. W. Yang, Nano Lett. 12, 1683 (2012).

[22] F. Krausz and M. Ivanov, Rev. Mod. Phys. 81, 163 (2009).

[23] S. Liu, M. Müller, Y. Sun, I. Hamada, A. Hammud, M. Wolf, and T. Kumagai, Nano Lett. 19, 5725 (2019).

[24] J. H. Yoon, F. Selbach, L. Schumacher, J. Jose, and S. Schlücker, ACS Photon. 6, 642 (2019).

[25] P. Liu, X. Chen, H. Ye, and L. Jensen, ACS Nano 13, 9342 (2019).

[26] L. Pierantoni, D. Mencarelli, and T. Rozzi, IEEE Trans. Microwave Theory Tech. 57, 1147 (2009).

[27] L. Pierantoni, D. Mencarelli, and T. Rozzi, IEEE Trans. Microwave Theory Tech. 56, 654 (2008).

[28] S. Ohnuki, T. Takeuchi, T. Sako, Y. Ashizawa, K. Nakagawa, and M. Tanaka, Int. J. Numer. Model.: Electron. Netw. Devices Fields 26, 533 (2013).

[29] I. Ahmed, E. H. Khoo, E. Li, and R. Mittra, IEEE Antennas Wireless Prop. Lett. 9, 914 (2010).

[30] K. Yabana and G. F. Bertsch, Phys. Rev. A 60, 1271 (1999).

[31] K. Yabana and G. F. Bertsch, Phys. Rev. B 54, 4484 (1996).

[32] Y. Kawashita, T. Nakatsukasa, and K. Yabana, J. Phys. Condens. Matter 21, 064222 (2009).

[33] M. A. L. Marques, A. Castro, G. F. Bertsch, and A. Rubio, Comput. Phys. Commun. 151, 60 (2003).

[34] C. Covington, D. Kidd, J. Gilmer, and K. Varga, Phys. Rev. A 95, 013414 (2017).

[35] T. Otobe, T. Hayashi, and M. Nishikino, Appl. Phys. Lett. 111, 171107 (2017).

[36] A. Crawford-Uranga, U. De Giovannini, E. Räsänen, M. J. T. Oliveira, D. J. Mowbray, G. M. Nikolopoulos, E. T. Karamatskos, D. Markellos, P. Lambropoulos, S. Kurth, and A. Rubio, Phys. Rev. A 90, 033412 (2014).

[37] K. Krieger, J. K. Dewhurst, P. Elliott, S. Sharma, and E. K. U. Gross, J. Chem. theory Comput. 11, 4870 (2015).

[38] Y. Li, S. He, A. Russakoff, and K. Varga, Phys. Rev. E 94, 023314 (2016).

[39] K. Yabana, T. Sugiyama, Y. Shinohara, T. Otobe, and G. F. Bertsch, Phys. Rev. B 85, 045134 (2012).

[40] M. Ruggenthaler, J. Flick, C. Pellegrini, H. Appel, I. V. Tokatly, and A. Rubio, Phys. Rev. A 90, 012508 (2014).

[41] M. Ruggenthaler, N. Tancogne-Dejean, J. Flick, H. Appel, and A. Rubio, Nat. Rev. Chem. 2, 0118 (2018).

[42] S. A. Sato, K. Yabana, Y. Shinohara, T. Otobe, K.-M. Lee, and G. F. Bertsch, Phys. Rev. B 92, 205413 (2015).

[43] S. A. Sato, Y. Shinohara, T. Otobe, and K. Yabana, Phys. Rev. B 90, 174303 (2014).

[44] X. Zhang, F. Wang, L. Jiang, and Y. Yao, Phys. Rev. B 95, 184301 (2017).

[45] I. Floss, C. Lemell, G. Wachter, V. Smejkal, S. A. Sato, X.M. Tong, K. Yabana, and J. Burgdörfer, Phys. Rev. A 97, 011401(R) (2018).

[46] N. Tancogne-Dejean, O. D. Mücke, F. X. Kärtner, and A. Rubio, Phys. Rev. Lett. 118, 087403 (2017).
[47] M. Lucchini, S. A. Sato, A. Ludwig, J. Herrmann, M. Volkov, L. Kasmi, Y. Shinohara, K. Yabana, L. Gallmann, and U. Keller, Science 353, 916 (2016).

[48] M. Schultze, K. Ramasesha, C. Pemmaraju, S. Sato, D. Whitmore, A. Gandman, J. S. Prell, L. J. Borja, D. Prendergast, K. Yabana, D. M. Neumark, and S. R. Leone, Science 346, 1348 (2014).

[49] G. Wachter, C. Lemell, J. Burgdörfer, S. A. Sato, X.-M. Tong, and K. Yabana, Phys. Rev. Lett. 113, 087401 (2014).

[50] S. He, C. Covington, and K. Varga, J. Appl. Phys. 123, 165102 (2018).

[51] J. Flick and P. Narang, Phys. Rev. Lett. 121, 113002 (2018).

[52] C. Schäfer, M. Ruggenthaler, H. Appel, and A. Rubio, Proc. Natl. Acad. Sci. USA 116, 4883 (2019).

[53] P. Wopperer, U. De Giovannini, and A. Rubio, Eur. Phys. J. B 90, 51 (2017).

[54] A. Russakoff, S. Bubin, X. Xie, S. Erattupuzha, M. Kitzler, and K. Varga, Phys. Rev. A 91, 023422 (2015).

[55] S. Bubin, M. Atkinson, K. Varga, X. Xie, S. Roither, D. Kartashov, A. Baltuška, and M. Kitzler, Phys. Rev. A 86, 043407 (2012).

[56] M. Šindelka, Phys. Rev. A 81, 033833 (2010).

[57] E. Lorin, S. Chelkowski, and A. Bandrauk, Comput. Phys. Commun. 177, 908 (2007).

[58] I. V. Tokatly, Phys. Rev. Lett. 110, 233001 (2013).

[59] J. Flick, M. Ruggenthaler, H. Appel, and A. Rubio, Proc. Natl. Acad. Sci. USA 112, 15285 (2015).

[60] C. Pellegrini, J. Flick, I. V. Tokatly, H. Appel, and A. Rubio, Phys. Rev. Lett. 115, 093001 (2015).

[61] R. Jestädt, M. Ruggenthaler, M. J. T. Oliveira, A. Rubio, and H. Appel, arXiv:1812.05049 (2018).

[62] K. Lopata and D. Neuhauser, J. Chem. Phys. 130, 104707 (2009).

[63] Y. Gao and D. Neuhauser, J. Chem. Phys. 137, 074113 (2012).

[64] Z. Hu, M. A. Ratner, and T. Seideman, J. Chem. Phys. 141, 224104 (2014).

[65] Y. P. Chen, W. E. Sha, L. Jiang, M. Meng, Y. M. Wu, and W. C. Chew, Comput. Phys. Commun. 215, 63 (2017).

[66] C. J. Ryu, A. Y. Liu, W. E. I. Sha, and W. C. Chew, IEEE J. Multiscale Multiphys. Comput. Tech. 1, 40 (2016).

[67] C. Xiang, F. Kong, K. Li, and M. Liu, IEEE J. Quantum Electron. 54, 1 (2018).

[68] T. Takeuchi, S. Ohnuki, and T. Sako, IEEE J. Quantum Electron. 50, 334 (2014).

[69] T. Takeuchi, S. Ohnuki, and T. Sako, Phys. Rev. A 91, 033401 (2015).

[70] K. S. Yee, IEEE Trans. Antennas Prop. 14, 302 (1966).

[71] R. Higdon, Math. Comput. 49, 65 (1987).

[72] S. Gedney, IEEE Trans. Antennas Prop. 44, 1630 (1996).

[73] G. Mur, IEEE Trans. Electromagn. Compat. EMC-23, 377 (1981).

[74] E. Runge and E. K. U. Gross, Phys. Rev. Lett. 52, 997 (1984).

[75] S. Ludwig, Ann. Phys. 327, 579 (1907).

[76] D. Kidd, C. Covington, Y. Li, and K. Varga, Phys. Rev. B 97, 024303 (2018).

[77] D. Kidd, C. Covington, and K. Varga, Phys. Rev. E 96, 063307 (2017).

[78] W. van Dijk and F. M. Toyama, Phys. Rev. E 90, 063309 (2014). 
[79] J. Muga, J. Palao, B. Navarro, and I. Egusquiza, Phys. Rep. 395, 357 (2004).

[80] D. E. Manolopoulos, J. Chem. Phys. 117, 9552 (2002).

[81] W. Kohn and L. J. Sham, Phys. Rev. 140, A1133 (1965).
[82] J. P. Perdew and A. Zunger, Phys. Rev. B 23, 5048 (1981).

[83] A. Russakoff, Y. Li, S. He, and K. Varga, J. Chem. Phys. 144 204125 (2016).

[84] M. Brack, Rev. Mod. Phys. 65, 677 (1993). 